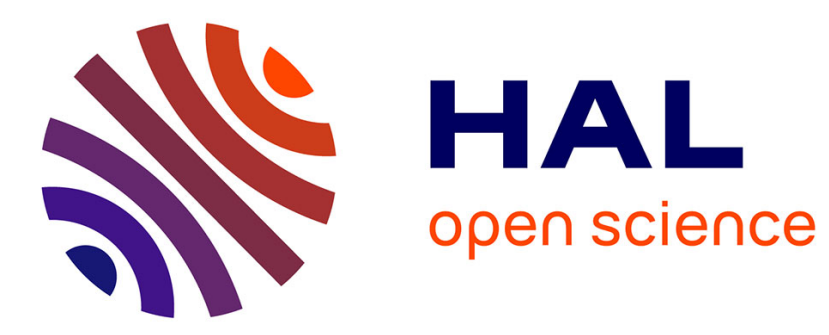

\title{
Les processus participatifs transforment-ils les représentations des élus ruraux sur leur rôle et leur territoire?
}

Nicolas Buclet, Danielle Salomon

\section{- To cite this version:}

Nicolas Buclet, Danielle Salomon. Les processus participatifs transforment-ils les représentations des élus ruraux sur leur rôle et leur territoire?. Espaces et sociétés (Paris, France), 2012, 3 (151), pp.123138. hal-00934829

\section{HAL Id: hal-00934829 \\ https://hal.univ-grenoble-alpes.fr/hal-00934829}

Submitted on 22 Jan 2014

HAL is a multi-disciplinary open access archive for the deposit and dissemination of scientific research documents, whether they are published or not. The documents may come from teaching and research institutions in France or abroad, or from public or private research centers.
L'archive ouverte pluridisciplinaire $\mathbf{H A L}$, est destinée au dépôt et à la diffusion de documents scientifiques de niveau recherche, publiés ou non, émanant des établissements d'enseignement et de recherche français ou étrangers, des laboratoires publics ou privés. 


\title{
LES PROCESSUS PARTICIPATIFS TRANSFORMENT-ILS LES REPRÉSENTATIONS DES ÉLUS RURAUX SUR LEUR RÔLE ET LEUR TERRITOIRE?
}

\author{
Nicolas Buclet ${ }^{1}$ et Danielle Salomon ${ }^{2}$ \\ Revue Espaces et sociétés
}

$2012 / 3-n^{\circ} 151$

pages 123 à 138

ISSN 0014-0481

\section{Résumé :}

L'article s'intéresse aux conséquences de la mise en échec des modalités traditionnelles de prise de décision, de l'évolution du rôle des élus, de la façon dont ils appréhendent leur territoire. Nous identifions dans un premier temps une tension entre la représentation que les élus se font de leur rôle et les aspirations au dialogue autour de projets de territoire. Nous voyons ensuite comment les promoteurs de mouvements de contestation pèsent sur le processus décisionnel et forcent les élus à négocier. Nous interrogeons enfin les conséquences des formes de dialogue engagées, en tant que vecteur potentiel d'apprentissage pour les élus, grâce au temps de réflexion associé au dialogue enclenché.

Mots-clef : participation, territoire, déchets ménagers, Ouest de la France, élu rural

\section{Abstract :}

This paper considers the consequences of traditional decision-making failures, the role of local decision-makers and their perception of the evolution of related territories. The paper starts from the existing tension between the way decision-makers perceive their own role and the demand of dialogue expressed by stakeholders. The paper shows how opponents to local authorities' projects weigh on the decision-making process and force local decision-makers to negotiate. Discussion is related to the consequences of the on-going dialogue, its apprenticeship role for decision-makers, notably due to the time of reflexion initiated.

Keywords: participation, territory, household waste, West of France, Rural Elected Representative

Les élus des différentes collectivités territoriales font l'expérience, à divers titres, d'une interpellation croissante autour de conflits d'usage et d'aménagement du territoire et plus

\footnotetext{
${ }^{1}$ Nicolas Buclet, Professeur à l'Institut d'urbanisme de Grenoble, Université Pierre Mendès-France, UMR 5194 PACTE nicolas.buclet@upmf-grenoble.fr ${ }^{2}$ Danielle Salomon, chercheuse associée au CSO (UMR 7116 CNRS-SciencesPo) dsalomon@noos.fr
} 
particulièrement de la part de riverains qui perçoivent que l'environnement modifié ou dégradé par un projet peut compromettre ou menacer leur santé. Ce phénomène est observable tant pour des sujets qui ne sont pas de leur compétence, au sens institutionnel du terme que pour des sujets qui relèvent de leurs compétences directes, comme celui des déchets municipaux. Dans tous les cas, l'interaction entre les élus et les riverains reste souvent difficile à établir de façon satisfaisante pour l'ensemble des parties et fait l'objet de tensions récurrentes.

Dans un travail concernant les élus locaux ou la gouvernance des territoires, Faure et Négrier (2007) interrogent la transformation des grandes métropoles et le regroupement par l'intercommunalité d'un grand nombre de fonctions. Cette transformation s'opère dans un contexte de décentralisation croissante des compétences, rôles et missions de l'Etat vers les collectivités territoriales. Il en découle en partie une professionnalisation des édiles politiques et de leurs adjoints administratifs. Avec ces mutations, plusieurs questions peuvent être soulevées entre différenciation ou standardisation des politiques, effets spécifiques des configurations territoriales, arguments rhétoriques liés à la proximité, à la territorialisation, à la gouvernance, associés ou non à des actions et des effets sensibles localement.

Le travail ici présenté part de l'observation de trois expériences de dialogue organisées par des collectivités territoriales ${ }^{3}$ en milieu à prédominance rurale autour de projets de traitement de déchets; il y est suggéré que les élus locaux font l'apprentissage d'une partie de ces transformations lorsqu'ils y sont confrontés et que des conflits surgissent avec les occupants des territoires. Autrement dit, l'apprentissage est forcé selon un tempo probablement différent en milieu rural qu'il ne l'est en milieu urbain.

Cet article ne revient ni sur les causes de la montée des mouvements contestataires, ni sur les formes, les modalités ou l'efficacité des dialogues ou des concertations menées dans les intercommunalités, deux problématiques déjà amplement illustrées et analysées dans d'autres travaux de recherche (Peribois et Roche, 2005 ; Renaud, 2001). Il s'agit ici d'apporter une contribution à la question des conséquences de cette mise en échec des modalités traditionnelles de prise de décision, tant sur l'évolution que cela suppose du rôle des élus, que sur l'évolution de l'appréhension de ce qui constitue le territoire concerné. Pour ce faire, nous mettons en évidence dans un premier temps, à partir des trois terrains d'observation, la tension existante entre la représentation que les élus se font de leur rôle et les diverses aspirations au dialogue autour de projets de territoire. Nous verrons en second lieu comment les acteurs promoteurs de mouvements de contestation ont les moyens de peser sur le processus décisionnel et de forcer les élus à entrer dans un processus de négociation. Nous interrogerons enfin les conséquences des formes de dialogue engagées, notamment en tant que vecteur potentiel d'apprentissage pour les élus, grâce au temps de réflexion associé au dialogue enclenché. L'observation des terrains amène à penser que, pour autant, ce potentiel n'a pas été pleinement appréhendé par les élus euxmêmes. Idéalement, ce temps de réflexion qui amène en particulier à nouer des relations avec des nouveaux partenaires, à concevoir autrement les flux de déchets dans le territoire, à comprendre ou au moins à reconnaître les demandes ou les appréhensions des populations, et ainsi apporter des modifications au projet initial, aurait pu déboucher pour les élus sur une nouvelle conception de leur rôle. Cette évolution n'est pas constatée sur nos terrains d'observation, les pratiques décisionnelles traditionnelles reprenant le dessus dans d'autres domaines et continuant d'incarner l'image de l'édile, élu pour représenter ses électeurs. L'on commence à envisager que la façon

\footnotetext{
${ }^{3}$ SITOM Ouest-Cornouailles (Finistère), SMICTOM des Châtelets (Côtes d'Armor), SIVERT Est-Anjou (Maine-etLoire).
} 
dont les élus se représentent leur rôle et leurs prérogatives se négocie en interaction avec leur capacité d'action et de décision.

\section{Contestation de projets de traitement des déchets et premières}

\section{conséquences sur le rôle des élus}

\section{Les territoires observés}

A partir d'une première observation des controverses portant sur des projets d'usines d'incinération (Buclet et al., 2003) l'Ouest de la France apparaissait comme particulièrement riche en expériences de concertation. En particulier, Saint Brieuc a été souvent citée pour avoir été la première ville à tenter une expérience de jury citoyen pour aborder la question des déchets (Barbier et al., 2009). Le travail de terrain mené en Maine-et-Loire, en Côte d'Armor et dans le Finistère nous a permis d'appréhender la question du face à face entre les élus et la question des déchets ménagers dans un contexte à dominante rurale, qualificatif qui peut paraître osé dans le cas du SMICTOM des Châtelets, qui comprend la ville de Saint Brieuc, mais qui reflète néanmoins bien ce qu'il nous a été donné d'observer. Rappelons en effet que la commune de Saint Brieuc n'a adhéré que tardivement à ce syndicat mixte, historiquement porté par des élus de zones rurales et périurbaines, caractéristique encore très présente au moment des débats, en 2003, ainsi qu'au moment des entretiens menés en 2007.

La population concernée par les syndicats mixtes concernés par l'étude est d'environ 210000 personnes dans le cas du SIVERT Est-Anjou (dont 60000 pour la communauté d'agglomération de Saumur), d'environ 50000 personnes dans le cas du SITOM Ouest-Cornouailles (autour de Douarnenez qui compte à peu près 15000 habitants) et d'environ 150000 personnes pour le SMICTOM des Châtelets (120 000 habitants pour l'agglomération briochine dont 50000 à Saint Brieuc).

Les trois territoires ont comme point commun, nous l'avons dit, d'avoir vécu des formes organisées de dialogue entre élus et population, procédures que les élus promoteurs ont souhaité afficher en tant que concertation, mais qui s'apparentent avant tout à de la consultation. En effet, si la population a été informée et si des réunions de débat, ouvertes à tous, ont été organisées, les élus locaux ont souhaité que le moment de la décision échappe à toute discussion participative. A l'instar de nombreux autres élus, nos élus font face à des mouvements de contestation des projets d'infrastructure de traitement des déchets, mais plus que d'autres ou, tout du moins, avant les autres, ils décident d'opter pour l'apaisement à travers le dialogue. Les mouvements d'opposition sont de nature sensiblement commune, avant tout portés par des associations dont certaines se sont constituées en réaction à l'émergence de projets d'incinération, voire de rumeurs de projets. Les entretiens menés nous montrent des élus soucieux de l'intérêt général dont ils s'estiment les garants. Cependant, quel que soit le terrain analysé, l'ensemble des élus n'est pas convaincu de l'opportunité de cette ouverture du processus de décision à la population et, de fait, à des associations contestataires. Les élus porteurs de la démarche seraient même plutôt minoritaires, comme en Ouest-Cornouailles où seul le président du SITOM, nouvellement élu, paraît déterminé à ouvrir les débats. C'est une position qui peut s'avérer inconfortable, les présidents des syndicats mixtes étant attendus au tournant par les élus qui suivent la démarche avec scepticisme. Pour certains, les opposants «n'ont qu'à se présenter aux élections s'ils veulent avoir leur mot à dire » (un élu local du Finistère). On reste donc dans un schéma assez classique 
d'élus pensant incarner la volonté générale du fait de leur mandat et n'entendant pas se dessaisir de leurs prérogatives.

Les élus interrogés se considèrent comme seuls porteurs de l'intérêt général. L'opposition entre les différentes visions de ce qui est «bien et juste» est tranchée avec les élections. Cette légitimité au nom d'un intérêt général local, longtemps disqualifiée par les porteurs de l'intérêt général national, s'est même renforcée dans les années 1970 avec le processus de décentralisation (Gaudin, 2007, p.203). Cette vision d'un intérêt général qui transcenderait les intérêts des individus ne porte plus. Dans le domaine de la gestion des déchets ménagers et assimilés, sur lequel repose notre analyse, les élus responsables de syndicats mixtes se voient contraints nolens volens, d'abandonner les modalités classiques de prise de décision et d'ouvrir en partie le processus aux populations locales et à ceux qui se présentent comme leurs représentants. Les échanges avec les publics sont une modalité d'affichage de la prise en charge d'une contestation, une façon de gagner du temps et une opportunité de gestion territoriale à laquelle se raccrocher face à une opposition frontale à des projets d'usines d'incinération.

La démocratie participative: une simple bouée de sauvetage qui dérive vers un débat sur le devenir des territoires

En ouvrant les débats sur l'avenir organisationnel et technique de la gestion locale des déchets ménagers, les élus n'avaient probablement pas imaginé ce qu'une telle expérience allait entraîner comme modifications de leur relation au territoire. Dans les trois cas analysés de façon plus détaillée, les élus s'étaient fait une représentation de l'incinération comme la «meilleure solution » disponible.

Dans le Maine-et-Loire, le contexte territorial se prêtait encore à une prise de décision sur un mode traditionnel. Les responsables du syndicat réunissaient un grand nombre d'atouts qui ont amplement contribué à faire aboutir leur projet. Les élus ont dès lors pu se limiter à une négociation sur les détails d'un projet d'usine d'incinération considéré par beaucoup comme incontournable. Dans le Finistère et dans les Côtes d'Armor, les élus se sentaient davantage affaiblis dans leur ancrage territorial. Les présidents respectifs des deux syndicats mixtes, nouvellement élus, souhaitaient sortir de l'impasse dans laquelle s'enfonçaient leurs prédécesseurs, les uns avec un projet d'incinérateur, les autres avec un projet d'usine de compostage dans un contexte agricole caractérisé par un excédent d'apport azoté. Repartir de zéro et faire participer le public à la discussion sur les orientations stratégiques territoriales en matière de déchets, leur a paru le moyen de contourner l'obstacle. Ce faisant, ces élus n'ont pas anticipé que les opposants au projet allaient profiter de l'occasion pour poser des questions allant au-delà de l'objectif des élus. Comme l'a montré Laurence Rocher (2006), les opposants à un projet d'incinération souhaitent discuter plus globalement des conséquences sur un territoire, sur son dynamisme économique, sur la capacité de ses habitants à se sentir partie prenante de son devenir, bref de l'intérêt commun ${ }^{4}$ qui peut se définir entre les occupants du territoire. Il y a en effet conflit entre des élus qui se sentent investis de la définition et de la mise en œuvre de l'intérêt général, et des acteurs qui contestent l'existence de cet intérêt général et souhaitent participer à la définition collective de l'intérêt commun. Cet affrontement est couramment

\footnotetext{
${ }^{4}$ En accord avec le rapport Brundtland (1987), nous préférons employer intérêt commun plutôt que bien commun. Avec l'intérêt commun, ressort non seulement l'idée du bien commun de la collectivité, mais également l'idée que chaque acteur peut ressortir potentiellement gagnant de la définition de ce qui pourrait être l'intérêt commun d'un territoire. Avec la dynamique créée, l'on ne considère plus nécessaire que l'individu se dépouille de son égoïsme au nom d'un intérêt général, mais qu'il contribue à un intérêt commun dont il bénéficiera en retour.
} 
observé et s'inscrit dans le cadre plus général tel qu'analysé par Laurent Davezies (2008) concernant l'existence de deux modèles de développement territorial, l'un portant sur la capacité des territoires à contribuer à la croissance nationale, l'autre sur une vision du territoire non pas comme facteur de production, mais comme support d'une population (Davezies, 2008, p.52). Le périmètre de la discussion dépasse de loin la simple question du traitement aval des déchets engendrés, pour s'étendre à un débat sur le mode de développement territorial à privilégier, notamment en lien avec des questionnements sur la consommation durable. C'est en partie la discussion autour de ce périmètre qui va déstabiliser des élus pour lesquels la question ne se posait même pas.

\section{Le poids des mouvements contestataires}

Pour prendre en charge les activités traditionnelles comme le traitement des déchets municipaux, les élus restent souvent prisonniers de la représentation traditionnelle qu'ils se font de leur mandat électif représentatif. Cette représentation est d'autant plus mise à mal qu'elle fait face à une complexité croissante des questions techniques et organisationnelles à traiter. La mobilisation d'acteurs opposés de facto à la façon traditionnelle de régler la question, et capables d'embrasser les enjeux de cette complexité croissante (à défaut d'en maîtriser la technicité sous toutes ces dimensions), constitue l'événement déclencheur d'une remise en cause du rôle des élus.

\section{Les acteurs contestataires avec qui compter}

La question des déchets, relativement simple à gérer jusqu'au tournant des années 1990, s'est transformée, à l'instar d'autres domaines de compétences des élus locaux, en un domaine nécessitant une expertise importante. La technicisation de la question pose quelques problèmes qui, s'ils ne sont pas traités en amont, renforceront la légitimité de mouvements d'opposition capables d'argumenter à partir des faiblesses des dossiers présentés par les élus. Si l'annonce d'un projet de traitement des déchets suscite l'émergence d'une contestation numériquement faible (quelques dizaines de personnes tout au plus sur les terrains analysés), l'efficacité de cette contestation s'appuie sur les faiblesses des projets. En effet, les mouvements contestataires peuvent eux-mêmes compter sur des ressources externes au territoire, reposant en grande partie sur l'expérience d'associations d'autres régions, sur l'existence de réseaux structurés et en particulier la fédération associative France Nature Environnement, mais également sur des liens tissés avec le CNIID (Centre National d'Information Indépendante sur les Déchets), association depuis longtemps mobilisée contre l'incinération. L'existence d'internet facilite substantiellement le travail d'identification de ces acteurs, et participe de l'élargissement du réseau d'acteurs mobilisés sur des espaces territoriaux différents mais autour d'une même problématique. Grâce à ce réseau les associations contestataires identifient des experts qu'elles invitent à s'exprimer devant des audiences numériquement intéressantes. Ces experts renforcent dans l'esprit des opposants, voire de personnes simplement inquiètes mais non mobilisées, la crainte de risques sanitaires, notamment en ce qui concerne l'incinération. Surtout, ils apportent du crédit aux informations véhiculées par divers groupes sociaux, que celles-ci proviennent de sources réputées comme sérieuses ou qu'elles reproduisent des rumeurs. Ce faisant, les membres des associations contestataires se forgent un argumentaire solidement étayé et, par leur engagement et leur investissement en acquisition de connaissance, acquièrent un statut de "sachant » face auquel seule une infime minorité d'élus (guère plus de un ou deux pour chacun des cas étudiés) peut 
faire face. Réciproquement, les élus sont informés des mouvements de mobilisation, des victoires obtenues dans d'autres sites et la presse spécialisée relaye les initiatives prises par leurs confrères dans divers lieux du territoire.

Comme cela est assez classique, nous avons également pu constater qu'au niveau local, la contestation s'inscrit dans l'histoire du territoire, des conflits et mobilisations passés qui expliquent les jeux d'alliance observables. Cette histoire doit être mise en parallèle avec celle du jeu entre élus, les relations privilégiées, les alliances objectives, les habitudes prises dans la répartition des rôles entre personnalités ou collectivités, de délégation de la confiance ou des décisions ou encore les styles de gouvernement. Ce jeu territorial politique, capable d'engendrer une maîtrise du dossier de la part des élus en Est-Anjou, sera davantage bousculé dans les deux cas bretons.

\section{Des mouvements qui interrogent les pratiques des élus et forcent la négociation}

L'émergence d'un mouvement de contestation est associée à des accidents de parcours dans la période de gestation des divers projets d'incinérateurs. A Lasse, alors même que la presse locale s'était fait l'écho de réflexions sur une future usine d'incinération porté par le SIVERT-Anjou, les habitants réalisent l'existence d'un projet suite au succès de la mobilisation autour d'un épandage de mâchefers provenant de l'ancien incinérateur effectué au bord d'une rivière, hors de tout respect de la réglementation. Dans le cas de la région Ouest-Cornouailles, des rumeurs de projets d'incinération circulent en différents lieux et se croisent avec l'émergence d'un projet de plate-forme de mâchefers qui n'aurait pas grand sens, comme le comprennent les riverains, s'il n'était pas couplé avec un incinérateur à proximité. Dans les Côtes d'Armor, le renoncement à l'agrandissement de la plate-forme de compostage, qui rencontre l'hostilité du milieu agricole, dans un contexte d'excès en apports azotés, fait émerger un projet d'incinération porté par le bureau du syndicat mixte et se voit relayé dans la presse. Bien que la décision ne soit pas ferme, le président du syndicat mixte affiche sa préférence a priori pour cette technique plutôt qu'une autre.

Dans les trois cas, riverains et associations manifestent leur hostilité parce qu'il s'agit d'incinération. Ils développent également une forte suspicion quant aux manœuvres des élus et les soupçonnent de dissimuler des faits (en ce qui concerne les retombées des mâchefers et les émissions de dioxine) et des débats et décisions prises en interne, plutôt que dans la transparence.

La mobilisation et le possible blocage du projet incitent les élus à ouvrir un débat avec le «public ». Néanmoins, la situation de départ entre l'Est-Anjou et les deux autres terrains n'est pas la même sur un certain nombre de plans :

- les efforts en faveur de la collecte sélective et du tri, préalables à l'annonce d'un nouveau projet d'infrastructure ont été plus poussés dans le Maine-et-Loire;

- la stabilité de l'équipe en place est forte au SIVERT, là où des présidents sont nouvellement élus en Bretagne issus d'une nouvelle majorité moins stable politiquement ;

- le SIVERT Est-Anjou s'intègre à un schéma départemental (PDEDMA) bien plus cohérent ;

- les gestionnaires de l'usine de Lasse (Maine-et-Loire) ont été plus vigilants en matière d'application de la réglementation que ceux de l'usine de Confort-Meilars (Finistère) ; 
- le SIVERT Est-Anjou s'organise à partir d'un bassin de population assez important numériquement pour rendre cohérent la construction d'une nouvelle usine d'incinération ;

- le monde associatif environnemental est beaucoup plus présent autour de Saint Brieuc qu'en Cornouailles et, davantage encore qu'en Anjou ;

- en Cornouailles, les populations d'origine urbaine (maisons secondaires, retraités), et autour de Saint Brieuc l'accroissement de l'étalement périurbain, contribuent à modifier les équilibres sociaux, contrairement aux alentours de Lasse où la population est relativement stable. La «demande » politique de la population évolue donc de façon moins marquée dans le Maine-et-Loire, tandis que l'on observe moins de conflits d'usage du territoire entre populations «de souche » et populations «mutantes » (Boyer et Orléan, 1994);

- contrairement aux cas bretons, le Sivert a derrière lui plusieurs années d'études, une instruction progressive et lente qui s'est faite avec et par le vote de l'unanimité des élus non seulement du syndicat, mais également de l'ensemble des élus du département.

C'est donc à partir de variables contextuelles assez différentes, que les élus ont fait évoluer les pratiques usuelles de prise de décision, et optent pour la concertation.

\section{Le rôle de l'expertise}

Il ressort en particulier de ces variables que le positionnement des élus du SIVERT Est-Anjou est plus stable politiquement et que l'équipe chargée du projet dispose d'un grand nombre de ressources et d'autonomie pour le conduire. Ce point est particulièrement visible lorsque l'on observe le type de relation que tissent les élus des syndicats mixtes avec les acteurs porteurs d'une expertise. A Lasse, le directeur administratif connaît intimement la directive-cadre qu'il a contribuée à négocier au ministère de l'Environnement. Le couple de dirigeants a reçu une délégation forte de la part des élus locaux et plusieurs cabinets ont été mobilisés pour contribuer à améliorer le dossier sur les différents plans, tandis que la coopération avec l'ADEME, la DRIRE et la DDASS ont été plus marquées qu'en Bretagne, dans un contexte de relations stables. La négociation avec les associations contestataires se fait donc à partir d'un projet solide, soutenu par l'essentiel des élus des différentes collectivités territoriales et construit à l'aide d'une expertise de qualité. Les responsables du syndicat mixte optent pour une stratégie de négociation compartimentée entre catégories d'acteurs, afin de ne pas favoriser des alliances de circonstance dans l'opposition au projet d'incinérateur. En Cornouailles comme dans les Côtes d'Armor, le recours à la concertation devient une issue possible au conflit, mais constitue d'abord une solution d'attente et de suspension de la décision. Les élus se sont lancés dans un projet insuffisamment mûri. En Cornouailles par ailleurs, le directeur des services techniques du SITOM, nouvellement embauché, fait apparaître que les chiffres du cabinet technique préalablement missionné ont été élaborés sur une base extrêmement discutable, dans le but de minimiser les coûts à la tonne d'un futur incinérateur. Enfin, la décision de recourir à la concertation est loin de faire l'unanimité au sein des élus du syndicat mixte.

Dans ces deux contextes, le recours à des cabinets d'études va être décisif quant à la forme que va prendre la concertation. Ces cabinets, spécialisés dans le montage et l'accompagnement de formes de concertation, vont proposer une méthode qu'ils revendiquent comme adaptée au contexte local, et qui donne le sentiment, surtout dans le cas briochin, qu'il s'agit d'expérimenter de nouvelles modalités de démocratie participative. 
Dans le cas du SITOM Ouest-Cornouailles, l'objectif du cabinet d'étude a surtout été de recréer les conditions d'un dialogue qui n'était plus possible entre élus et opposants. La formule retenue ressemble surtout à une procédure de consultation, dans la mesure où, tant en phase de préparation qu'au moment des différentes réunions publiques, l'objectif était de recueillir l'avis de la population et du monde associatif. Des scenarii (un premier fondé sur la valorisation énergétique maximale, un deuxième sur la valorisation maximale des déchets organiques et un troisième reposant sur un recours plus important au stockage des déchets) ont été élaborés à l'aide d'un cabinet technique, afin de présenter une base de discussion pour les débats publics. Le choix du cabinet de conseil technique a cependant créé un biais informationnel en faveur de l'incinération, du fait des compétences du consultant. Il ne s'agit pas d'un choix délibéré, mais de l'incapacité, reconnue par le consultant lui-même, d'apporter autant d'éléments techniques sur les alternatives à l'incinération, faute de compétences aussi approfondies que pour cette dernière technique. Cet aspect a joué sur l'interprétation des intentions du SITOM qu'en ont fait les associations, le biais en faveur de l'incinération n'étant qu'une preuve de plus, si besoin était encore, que la «concertation » n'avait été organisée que comme outil tactique afin d'affaiblir l'opposition au projet de Confort-Meilars. La série de réunions publiques, tenue sur une période d'à peine plus d'un mois, a donc surtout été l'occasion pour les associations les plus contestataires de prendre la parole et d'exprimer, parfois de façon virulente, leur point de vue en pleine opposition avec l'a priori des élus en faveur de l'incinération. Ces échanges de type conflictuel ont surpris le rare public présent, peu au fait des enjeux et étonné que l'on interagisse de la sorte avec les élus, plutôt que sur le mode de la confiance. L'expertise technique mobilisée par le SITOM s'est montrée en outre trop mince face au degré de connaissances acquis par les associations contestataires. Ainsi, contrairement à ce qui a été observé en Maine-et-Loire, le processus n'a pas été toujours bien maîtrisé par les élus, donnant encore plus aux associations contestataires le sentiment de détenir la meilleure expertise. De leur point de vue, les élus se sont sentis légitimés par « la meilleure » proposition face à d'autres acteurs peu ou mal renseignés sur les options possibles en matière de gestion des déchets.

Dans le pays briochin, ayant reculé une première fois face au monde agricole, les élus voyaient dans l'incinération le moyen de résoudre leur problème. L'opposition structurée, et accompagnée dans son mouvement par la presse, a vite fait comprendre qu'un tel projet ne se ferait pas sans mal. Le directeur des services techniques, ancien ingénieur de l'ADEME, a alors joué un rôle déterminant dans la suite des opérations en suggérant un certain nombre d'éléments :

- l'achat du terrain à proximité de l'usine de compostage existante, afin de disposer du foncier nécessaire à la future infrastructure de traitement ;

- les élus ne sont pas tous convaincus de la nécessité de se lancer dans la construction d'un incinérateur. Un débat paraît nécessaire ;

- l'organisation d'un débat public sur la question permettant d'ouvrir au dialogue avec les parties prenantes. L'idée n'est pas de cautionner l'incinération, selon le directeur technique, mais de bénéficier des idées échangées.

Le point de départ du débat public est la constitution d'un comité de pilotage dont l'objectif premier est de définir la méthode. Le choix du cabinet de conseil devait éviter que ne s'impose une méthode «clé en main» provenant d'un cabinet «parisien » qui ne s'intéresserait pas au contexte local. La structure choisie devait donc être légère afin de s'adapter aux orientations affirmées par le comité de pilotage. La procédure qui ressort des réflexions du comité de pilotage 
et de l'interaction avec le cabinet d'étude est un hybride entre la formule de la conférence de consensus et celle du débat public. Nous avons vu par ailleurs (Barbier et al., 2009) combien le panel de citoyens a été impliqué en première ligne dans les débats avec le public, tandis que le cabinet d'étude a influencé la teneur de l'expertise avec une liste « d'experts » particulièrement peu favorable à l'incinération. C'est donc sans surprise que les recommandations issues du panel de citoyens éliminent cette solution technique.

\section{Les conséquences du processus de négociation sur le rôle des élus}

Il nous reste à analyser les conséquences des formes de dialogue adoptées sur le rôle des élus. Déterminante pour l'apprentissage des élus est la question du temps, de la suspension de la décision en lien avec l'émergence du conflit et de son mode de traitement.

\section{Les effets de la suspension de la décision : le temps du mûrissement}

Si le SIVERT Est-Anjou a longuement préparé son projet, prenant ainsi le temps de la réflexion avant que celui-ci ne fasse son apparition dans l'arène publique, le temps des négociations a principalement abouti à la mise en place d'un dispositif de surveillance de la qualité de l'environnement, objet conventionnel apte à créer un consensus suffisant autour de l'action publique. Dans les deux cas bretons le suspens du temps de la décision, lors du déroulement de la concertation, a été source de réflexion quant au cadrage du problème (Mormont, 2006) et à l'univers des possibles techniques, organisationnels et institutionnels. Ainsi, en Cornouailles, le directeur des services techniques du SITOM admet que le temps de réflexion imposé par la préparation de l'exercice de consultation du public leur a permis de s'interroger plus longuement quant aux options possibles, mais également de comprendre que le recours à l'incinération constituait une charge financière excessive pour un si petit syndicat (moins de 100000 habitants en habitat dispersé). Par ailleurs, tant à Saint Brieuc qu'en Cornouailles, les élus ont appris que le contenu de leur mandat ne les condamnait pas pour autant à trouver une solution à la gestion des déchets sans même envisager de changement d'échelle territoriale, aspect qui avait été pris en compte au préalable par les élus du SIVERT Est-Anjou. A Saint Brieuc, il apparaît progressivement aux yeux des élus, grâce au débat et à la controverse, que l'échelle pertinente de réflexion ne se limite pas au périmètre du SMICTOM des Châtelets, mais englobe la quasi-totalité du département des Côtes d'Armor. Ce changement d'échelle a été rendu possible, du fait que les élus ont accepté l'invitation du panel de citoyens d'investir le champ de la réduction des flux de déchets, notamment organiques, nécessitant un traitement par incinération ou enfouissement. En Cornouailles, la solution qui se dessine, puis deviendra effective près de cinq ans après le débat, est celle d'une fusion du syndicat mixte avec une structure analogue voisine, et disposant elle-même d'un outil d'incinération disproportionné par rapport à ses seuls besoins. Dans les deux cas bretons, la contestation, le temps apparemment perdu du fait du dialogue rendu nécessaire pour désamorcer politiquement le terrain, aboutira finalement dans les années qui suivent à l'émergence de solutions cohérentes à une échelle territoriale plus large. 


\section{Quel apprentissage de la part des élus?}

L'on peut s'interroger pour savoir si ce type d'expériences qui s'inscrivent dans la durée et aboutissent à des transformations des projets modifient les représentations que se font les élus locaux du contenu de leur mandat et avec elles, leur façon de construire les politiques publiques : Se produit-il un effet d'apprentissage des effets de la démarche suivie allant au-delà du domaine des déchets ? Cessent-ils de résoudre des problèmes au cas par cas dans un cadre territorial préconstitué et indiscutable, pour relier toute décision à une vision élargie du territoire et de l'environnement tenant compte des aspirations de ses occupants en termes de qualité de vie ? Cette vision pourrait aboutir à un élargissement de la scène d'action, à l'incorporation de préoccupations portées par des riverains, associations, ou encore professionnels de la santé. Cette transformation amènerait les élus, confrontés à d'autres décisions publiques, à d'autres façons de concevoir de nouveaux projets de territoire,

Sans invalider cette hypothèse, qu'il serait intéressant de mettre à l'épreuve sur d'autres terrains, cet apprentissage ne s'est pas produit sur les terrains analysés, et notamment dans les deux cas bretons, là où pourtant le dialogue a porté sur l'ensemble de la politique de gestion des déchets à mener dans les années à venir. Plus lourd de conséquence, le fait qu'aucun investissement de poids, permettant de symboliser l'action, de la rendre visible, n'ait été lancé dans la foulée du processus participatif, a contribué à affaiblir auprès de nombre d'acteurs la perception d'une quelconque utilité de semblables démarches de dialogues. Pour l'importante fraction des élus hostiles au processus ou, pour le moins, perplexes face à cette nouvelle façon de décider, le décalage entre les débats instaurés et la décision prise a, de leur point de vue, confirmé leur position et leur a donné des arguments supplémentaires (gaspillage d'argent public). Bien qu'il ne soit pas possible d'établir un lien de causalité avec le dossier des déchets, on peut constater que l'équipe municipale de Douarnenez sortante n'a pas bénéficié d'un effet électoral substantiel. Elle a été battue aux élections suivantes de 2008, excluant de fait le président du syndicat mixte de sa fonction. En revanche, dans l'Est-Anjou les élus sont sortis renforcés de l'exercice, puisque l'incinérateur s'est construit et que le président du SIVERT est parvenu à maintenir autour de sa démarche l'essentiel des élus. S'agissant des élus favorables au processus participatif, en Bretagne la dureté des débats, les attaques subies, y compris sur un plan personnel, de la part de certains représentants du monde associatif, n'ont pas contribué à les inciter à recommencer. A Saint Brieuc notamment, alors même que plusieurs membres du panel de citoyens se disaient prêts à poursuivre leur engagement en collaboration avec les élus, ces derniers ont préféré tourner au plus vite la page d'un épisode éprouvant. Lorsque la question leur est posée des raisons les poussant à renoncer à des formes innovantes de dialogue, le coût de la démarche est bien souvent évoqué. Sans doute que le retour sur investissement politique n'a pas été à la hauteur des attentes. Depuis plusieurs décisions prises l'ont été sur la base du modèle décisionnel traditionnel.

\section{Un territoire modifié}

Si l'on s'intéresse de plus près aux deux cas bretons, on se retrouve dans une situation qui peut paraître paradoxale. La page semble tournée sur les démarches participatives qui ont pourtant joué un rôle en favorisant une réflexion de fond sur ce qu'il convenait de mettre en œuvre en matière de gestion des déchets. Malgré cela, le jeu d'acteur caractérisant le territoire s'en trouve modifié. Les intercommunalités définies à partir d'équilibres et d'affinités politiques n'apparaissent plus comme l'espace incontournable, le périmètre indépassable au sein duquel

gérer la question. La technicisation de la question des déchets aurait dû faire comprendre aux élus 
qu'il leur fallait changer d'échelle, sortir d'un périmètre trop restreint eu égard aux flux de production, de circulation et de valorisation des déchets, mais également pour des raisons techniques et financières. Grâce aux processus de dialogue instaurés par les syndicats mixtes, les mouvements contestataires ont permis de mettre en évidence de telles carences, ou tout du moins ont contraint les élus à en saisir l'importance. Les modalités techniques demeurent tributaires du dimensionnement possible des infrastructures, de la dispersion ou la concentration sur le territoire, mais aussi des possibilités de valorisation complémentaires aux sites de traitement.

En Cornouailles, l'épisode contestataire et sa gestion par le dialogue a également fait comprendre aux élus qu'ils ne pouvaient se contenter de dépendre d'une expertise externe au territoire. L'assistance est une chose, la dépendance une autre, qui ne permet pas aux élus de saisir les tenants et aboutissants du dossier. En ce sens, le territoire se recompose. Les élus semblent avoir compris l'importance de ressources cognitives internes et se sont dotés de moyens supplémentaires pour accroître la capacité du territoire à se penser et à appréhender les questions techniques qui s'imposent à lui. En d'autres termes, comme dans nombre d'autres domaines (Gumuchian et Pecqueur, 2007), les élus comprennent l'importance de doter le territoire de ressources qui lui soient propres.

Le troisième changement important issu de l'épisode contestataire a trait aux transitions démographiques et aux phénomènes migratoires qui entraînent des représentations distinctes ou entrant en concurrence entre elles. Cela a été dit, les contextes bretons se distinguent du contexte de l'Est-Anjou par l'arrivée numériquement importante de nouvelles populations, en zone périurbaine comme à Saint Brieuc ou en zone rurale comme en Cornouailles (retraités, résidences secondaires). Le territoire s'en trouve modifié par l'émergence : d'un rapport différent à l'élu local que l'on connaît moins au départ, avec qui l'on ne partage pas d'histoire commune ; d'importation de cultures politiques en rupture avec les habitudes forgées par les acteurs traditionnels du territoire ; de modes de vie urbains et d'une représentation différente de la vie en milieu rural ${ }^{5}$ et de la place des activités humaines en relation avec l'écosystème.

Notre travail de terrain et d'analyse, financé par l'Ademe, s'est achevé fin 2008, soit environ cinq ans après la tenue des initiatives participatives analysées dans cet article. Si des conclusions provisoires peuvent être tirées de ce travail, il nous est cependant difficile de comprendre l'épaisseur et la résilience des modifications observées. À ce titre, il serait pertinent que de nouveaux travaux, touchant à ces mêmes territoires, soient menés afin d'interroger l'impact à plus long terme des expériences analysées dans cet article sur la recomposition du jeu d'acteur et des représentations que se construisent les acteurs de leur espace de vie.

\section{Conclusion}

Il y a quelque part un sens d'inachevé des expériences participatives menées, en particulier dans les deux cas bretons. En revanche, une démarche qui s'assimile avant tout à une négociation (plus qu'à une concertation malgré l'usage facile fait de cette notion) menée au travers d'échanges maîtrisés par des partenaires particulièrement puissants, a pu aboutir tout en connaissant des transformations. Que faut-il en conclure ? La complexité des variables techniques, sociales et financières à prendre en compte, sur des dossiers considérés autrefois comme simples, accroît la capacité d'autres acteurs du territoire à contester la compétence des élus locaux non seulement en

\footnotetext{
${ }^{5}$ Cet aspect est particulièrement ressorti des entretiens en Cornouailles et, dans une moindre mesure en Est-Anjou.
} 
termes cognitifs mais aussi par des actions concrètes. La solidité de l'assise des élus, mais également la capacité à anticiper cette contestation en présentant à la population un dossier soutenu par une expertise technique et organisationnelle forte, a caractérisé le cas du Maine-etLoire et a manqué dans les deux cas bretons, par ailleurs terres de contestation et de compétition entre visions éloignées quant au devenir du territoire. Comme cela a été dit par ailleurs (Barbier et al., 2009), il faut distinguer l'impact des processus enclenchés sur les décisions prises et sur l'évolution des pratiques des élus. $\mathrm{Si}$, avec près de six ans de recul, les choix de gestion des déchets semblent cohérents, la question de l'apprentissage des élus en termes de gouvernance et de représentation du territoire dans lequel s'inscrit leur action, est bien plus contrastée. Les élus bretons, pourtant à l'origine d'initiatives novatrices, ne semblent guère empressés de retenter l'expérience. Certains éléments nous mènent cependant à penser que le territoire ne pourra plus être pensé de la même façon par les acteurs impliqués, y compris par les élus. En effet, à partir des entretiens menés dans le cadre de ce travail, il est possible de comprendre que le jeu d'acteurs observé n'est en rien spécifique à la question de la gestion des déchets. D'autres projets territoriaux ont été évoqués (aménagements routiers en particulier) autour desquels les acteurs se structurent et s'organisent de manière assez semblable. Les élus, échauffés par les attaques subies, n'ont pas souhaité reproduire la démarche participative que nous avons analysée. À en croire Maurice Leblanc (2006) les tensions observables sur nos terrains d'analyse se retrouvent en France à un niveau général, dès lors que des élus s'investissent et mettent en place des dispositifs participatifs. Selon cet auteur, trois légitimités s'affronteraient au cours de ces moments de dialogue: le suffrage universel, l'expertise et la mobilisation des habitants concernés. Reste une question à se poser, qui mériterait de nouvelles investigations de terrain, afin de comprendre si les tensions observables sont, comme le pense Maurice Leblanc (2006) le produit de la démarche entreprise, ou au contraire si la démarche participative est l'occasion pour des tensions territoriales fortes de remonter à la surface. En ce cas, la démarche participative pourrait également être vue comme l'exutoire à ces tensions, moment certes particulièrement inconfortable pour les élus, mais moment nécessaire afin de permettre aux acteurs d'élaborer une vision commune des enjeux territoriaux.

\section{Bibliographie}

Barbier, R. ; Bédu, C. ; Buclet, N. 2009 «Quand les «modernes » font l'expérience de la «liberté des anciens ». Leçons du jury citoyen de Saint-Brieuc ». Politix, n 86, p.189-207.

Boyer, R ; Orléan, A. 1994. «Persistance et changement des conventions. Deux modèles simples et quelques illustrations ». In A.Orléan (dir.). Analyse économique des conventions. Paris, PUF, p.219-247.

Brundtland G. H., 1987. Notre avenir à tous. Rapport de la Commission mondiale sur l'environnement et le développement de l'ONU.

www.cannes.com/images/stories/docs/qualitevie/environnenment/dev_durable/rapport_brundtlan d.pdf.

Buclet, N. (dir.) ; Bourg, D. ;Gilotte, L. 2003. Impact du risque technologique sur la stabilité institutionnelle d'un système organisationnel : le rôle de l'incinération dans la gestion des déchets ménagers et assimilés, Programme Evaluation et Prise en Compte des Risques Naturels et Technologiques, Ministère de l'Ecologie et du Développement Durable.

Buclet, N. ; Salomon, D. 2008. Influence de la démocratie participative sur la représentation sociale des risques liés à la gestion des déchets. Rapport final pour l'ADEME. 
Davezies, L. 2008. La République et ses territoires : la circulation invisible des richesses. Paris. Seuil. Collection La République des Idées.

Faure, A. ; Négrier E. (dir.). 2007. Les politiques publiques à l'épreuve de l'action locale. Critiques de la décentralisation, Paris, L'Harmattan.

Gaudin, J-P. 2007. Gouverner par contrat. Paris. Sciences Po Les Presses. $2^{\text {ème }}$ édition revue et augmentée.

Gumuchian, H. ; Pecqueur, B.(dir.), 2007, La ressource territoriale, Economica, Collection Anthropos, Paris.

Leblanc M., 2006. «Conflits et transactions sociales : la démocratie participative n’est pas un long fleuve tranquille ». Sciences de la Société. nº9, p.24-37.

Mormont, M. 2006. «Conflit et territorialisation ». Géographie, économie, société. Vol.8, p.299318.

Peribois, C., Roche, S. 2005. «L'apport de l'information géographique à la construction des intercommunalités : les défis de la concertation en France et en Belgique », Annales de la recherche urbaine, $\mathrm{n}^{\circ} 99, \mathrm{p} .85-92$.

Renaud, Y. 2001. « De la contestation à la concertation », Annales de la recherche urbaine, $\mathrm{n}^{\circ} 89$, p.62-69.

Rocher, L. 2006. Gouverner les déchets: gestion territoriale des déchets ménagers et participation publique. Thèse de doctorat en Aménagement de l'espace soutenue le 20 novembre 2006 sous la direction de C. Larrue à l'Université François Rabelais - Tours. 